\title{
Graham MS Dann et Giuli Liebman Parrinello (dir.), The Sociology of Tourism. European Origins and Developments
}

Tourism Social Sciences Series, vol. 12, Emerald, 2009, 480 pages

\section{Bertrand Réau}

\section{(2) OpenEdition}

Édition électronique

URL : http://journals.openedition.org/tourisme/497

DOI : 10.4000/tourisme.497

ISSN : 2492-7503

Éditeur

Éditions touristiques européennes

\section{Édition imprimée}

Date de publication : 1 décembre 2011

Pagination : 117-118

ISSN : 2109-5671

\section{Référence électronique}

Bertrand Réau, « Graham MS Dann et Giuli Liebman Parrinello (dir.), The Sociology of Tourism. European Origins and Developments », Mondes du Tourisme [En ligne], 4 | 2011, mis en ligne le 30 septembre 2015, consulté le 22 septembre 2020. URL : http://journals.openedition.org/tourisme/497 ; DOI : https:// doi.org/10.4000/tourisme.497

\section{Ce document a été généré automatiquement le 22 septembre 2020}

\section{cc) (1)}

Mondes du tourisme est mis à disposition selon les termes de la licence Creative Commons Attribution - Pas d'Utilisation Commerciale - Pas de Modification 4.0 International. 


\section{Graham MS Dann et Giuli Liebman Parrinello (dir.), The Sociology of Tourism. European Origins and Developments}

Tourism Social Sciences Series, vol. 12, Emerald, 2009, 480 pages

\section{Bertrand Réau}

\section{RÉFÉRENCE}

Graham MS Dann et Giuli Liebman Parrinello (dir.), The Sociology of Tourism. European Origins and Developments, Tourism Social Sciences Series, vol. 12, Emerald, 2009.

Disons-le d'emblée, The Sociology of Tourism n'est pas un ouvrage de sociologie du tourisme, mais bien une contribution à une histoire sociale des sciences sociales du tourisme (comme en atteste le sous-titre). C'est bien là que réside l'intérêt de ce volume.

Publié en 2009, cet ouvrage collectif sur les origines et le développement européens de la sociologie du tourisme réunit quatorze contributeurs et traite d'une dizaine de pays (ou d'aires linguistiques) : les pays de langue allemande, la France, la Belgique et les Pays-Bas, la Scandinavie, la Grèce, l'Italie, l'ex-Yougoslavie, la Pologne et l'Espagne. Les coordinateurs commencent, en introduction, par justifier le choix d'un tel volume sur l'Europe : alors que les sciences sociales sur le tourisme sont largement dominées par le monde anglo-saxon, tant au regard de la quantité que des revues et des associations spécialisées sur cet objet, la contribution scientifique des pays européens a largement été négligée. Il y a là, bien évidemment, un effet dû à la domination linguistique : la traduction en anglais de textes souvent rédigés dans la langue natale des auteurs vise à diffuser plus largement ces recherches, trop souvent méconnues, des anglophones. Ils 
se posent ensuite la question de la construction nationale (et ici européenne) de cette spécialité, des rapports de la sociologie du tourisme à la sociologie générale, de la sociologie du tourisme à la sociologie des loisirs, des frontières disciplinaires et des questions de traduction. Ils retracent ensuite rapidement les grandes évolutions (auteurs, théories dans différents pays) de cette spécialité de la fin du XIX ${ }^{e}$ siècle à nos jours. Enfin, ils concluent avec un focus sur chaque contributeur.

Chaque chapitre correspond ensuite à des exposés sur les "traditions nationales". Le lecteur y trouvera d'importantes informations sur les spécificités théoriques et institutionnelles. En ce sens, l'ouvrage offre une première introduction synthétique des différentes histoires nationales de la sociologie du tourisme. Néanmoins, en dépit des questions importantes posées en introduction, l'ensemble reste très descriptif et très peu réflexif. Pourtant, la sociologie des sciences a connu ces dix dernières années d'importants développements qui auraient permis de soumettre à analyse ce qui est souvent pris comme des faits. Par exemple, dans le chapitre qui porte sur la France, Marie-Françoise Lanfant décrit l'émergence d'une sociologie des loisirs fonctionnaliste dans les années 1960-1970, mais elle ne met jamais en perspective ce courant avec le développement général de la discipline en France. Ces décennies sont pourtant marquées par des avancées scientifiques incontournables. Simple exemple: la publication de La Distinction, en 1979, participe à reconfigurer la sociologie de la culture et certainement à reléguer au second plan la sociologie des loisirs proposée par Joffre Dumazedier. Or, comment comprendre le développement d'un courant sociologique sans le replacer systématiquement dans l'espace académique auquel il appartient? Comment expliquer le succès puis le déclin de l'approche de Dumazedier et de MarieFrançoise Lanfant sans prendre en compte les transformations du champ des sciences sociales, et plus particulièrement de celles qui prennent le tourisme pour objet? Fort heureusement, tous les chapitres ne sont pas aussi aveugles aux évolutions récentes de la sociologie du tourisme que celui consacré à la France. Il faudrait s'interroger sur cette spécificité française. En effet, pour un ouvrage publié en 2009, Marie-Françoise Lanfant arrête son histoire, largement autocentrée, quasiment dix ans auparavant. C'est pourtant au tournant des années 2000 que les travaux sur le tourisme en sociologie, mais aussi en anthropologie, prennent une nouvelle orientation qui se concrétise par la publication de numéro de revues généralistes et reconnues par ces disciplines (à la différence des publications des trente années précédentes). La construction d'objets de recherches moins "tourismo-centrés" (et par là même susceptibles d'intéresser au-delà des spécialistes du tourisme), le renouvellement des problématiques et des terrains d'enquêtes ne sont pas étrangers à ce changement.

S'il ne propose en rien un travail de réflexivité nécessaire à toute avancée scientifique, ce livre réunit des témoignages précieux sur un courant de sociologie. Il y a là une invitation à mener une sociologie des sciences sociales, en s'interrogeant notamment sur les conditions sociales de transformation des recherches sur le tourisme. 